\title{
INVESTIGATION OF FASCICULATION AND ELONGATION PROTEIN $\zeta-1$ (FEZ1) IN PERIPHERAL BLOOD REVEALS DIFFERENCES IN GENE EXPRESSION IN PATIENTS WITH SCHIZOPHRENIA
}

\author{
Vachev $\mathrm{TI}^{1,2}$, Stoyanova VK ${ }^{2, *}$, Ivanov $\mathrm{HY}^{2}$, Minkov IN ${ }^{1}$, Popov $\mathrm{NT}^{3}$
}

\begin{abstract}
*Corresponding Author: Associate Professor Vili K. Stoyanova, M.D., Ph.D., Department of Pediatrics and Medical Genetics, Medical University - Plovdiv, 15A Vasil Aprilov St., 4000 Plovdiv, Bulgaria. Tel: +359-32602-431; Fax: +359-32-602-593. E-mail: vilsto@abv.bg
\end{abstract}

\begin{abstract}
Schizophrenia (SZ) is a chronic neuropsychiatric disorder characterized by affective, neuromorphological and cognitive impairment, deteriorated social functioning and psychosis with underlying molecular abnormalities, including gene expression changes. Observations have suggested that fasciculation and elongation protein $\zeta-1$ (FEZ1) may be implicated in the pathogenesis of schizophrenia. Nevertheless, our current knowledge of the expression of FEZl in peripheral blood of schizophrenia patients remains unclear. The purpose of this study was to identify the characteristic gene expression patterns of FEZI in peripheral blood samples from schizophrenia patients. We performed quantitative reverse-transcriptase (qRT-PCR) analysis using peripheral blood from drug-free schizophrenia patients $(n=29)$ and age and gender-matched general population controls $(n=24)$. For the identification of FEZ1 gene expression patterns, we applied a comparative threshold cycle (CT) method. A statistically significant difference of FEZI mRNA level was revealed in schizophrenia subjects compared to healthy controls $(p=0.0034)$. To the best of our knowledge, this study is the first describing a down-regulation of FEZ1 gene expression in
\end{abstract}

\footnotetext{
${ }^{1}$ Department of Plant Physiology and Molecular Biology University of Plovdiv "Paisii Hilendarski”, Plovdiv, Bulgaria

${ }^{2}$ Department of Pediatrics and Medical Genetics, Medical University - Plovdiv, 4000 Plovdiv, Bulgaria

${ }^{3}$ State Psychiatry Hospital Pazardzk, Pazardzk, Bulgaria
}

peripheral blood of patients with schizophrenia. Our results suggested a possible functional role of FEZ1 in the pathogenesis of schizophrenia and confirmed the utility of peripheral blood samples for molecular profiling of psychiatric disorders including schizophrenia. The current study describes FEZ1 gene expression changes in peripheral blood of patients with schizophrenia with significantly down-regulation of FEZ1 mRNA. Thus, our results provide support for a model of SZ pathogenesis that includes the effects of FEZ1 expression.

Keywords: Fasciculation and elongation protein $\zeta-1$ (FEZ1); gene expression; quantitative reversetranscriptase polymerase chain reaction (qRT-PCR); schizophrenia (SZ)

\section{INTRODUCTION}

Schizophrenia (SZ) is a severe mental disorder affecting approximately $1.0 \%$ of the human population worldwide [1]. Twin, family and adoption studies strongly support that genetic factors play an important role in the etiology of SZ. Currently, numerous genetic studies, including linkage scans and their meta-analyses, candidate gene association analyses, gene expression and genome-wide association studies (GWAS) have identified particular genes and chromosomal loci with the disorder [2-4]. Circulating blood is easily accessible material and has been suggested as an alternative to tissue samples for molecular profiling of human disease and disease risk [3,5]. This is based on the capacity of peripheral blood to reflect pathological 
changes in the body. As the brain tissue is not easily accessible for investigation, blood-based expression profiling is increasingly being undertaken to search potential biomarkers for SZ [6,7]. Fasciculation and elongation protein $\zeta-1$ (FEZ1) is one of the first identified binding partners of Disrupted-in Schizophrenia 1 (DISC1), a susceptibility gene for major mental disorders including SZ in yeast two-hybrid screen of an adult human brain library [8]. Moreover, proteomic techniques revealed that $F E Z 1$ protein interacts with various intracellular partners, such as motor signaling, and structural proteins, one of which is DISC1 [9]. Currently, the role of FEZ1 in mammalian neuronal development in vivo is not well understood. The FEZ1 null mice exhibit hyperactivity and enhanced responsiveness to psychostimulants [10], supporting a po-tential contribution of FEZ1 dysfunction to SZ. Recently, additional evidence based on sequencing of DISCl-interacting partner genes including FEZ1 revealed an increased burden of rare missense variants in SZ susceptibility in an isolated northern Swedish population [11]. In contrast, the FEZ1 gene shows no association to SZ in Caucasian or African American populations [12]. However, no association was found in either population between specific haplotypes and any of the psychiatric disorders [13]. Interestingly, there is a significant reduction of FEZ1 mRNA in both hippocampus and dorsolateral prefrontal cortex of SZ patients and also an association of the DISC1 genotype and FEZ1 mRNA levels [9]. All these findings raise the possibility that $F E Z 1$ and DISC1 may assist in regulating both neuronal development and risk for SZ. We hypothesized that previously shown FEZ1 mRNA levels in prefrontal cortex of SZ patients may be relevant to those in peripheral blood tissue. The aim of this study was to identify gene expression patterns of FEZ1 in peripheral blood samples from SZ patients.

\section{MATERIALS AND METHODS}

Ethics Statement. This study and the informed consent forms were approved by the Medical University of Plovdiv Ethics Committee.

Participants. Written informed consent was obtained from 29 patients recruited at the State Psychiatry Hospital Pazardjik, Pazardzk, Pazardzk, Bulgaria and 24 healthy volunteers. Routine psychiatric examination, wide medical hystory and the Mini-
International Neuropsychiatric Interviews were done by a certified psychiatrist to evaluate the diagnosis of paranoid SZ only, on Diagnostic and Statistical Manual of Mental Disorders (IVth edition) criteria and to exclude any mental disorder in the controls. Important inclusion criteria were that the participants had not received any medication (even psychotropic) 1 month before blood sampling and they had a standard breakfast, so they were assessed in a state of exacerbation. Persons with other chronic medical and current acute somatic/neurologic illness, alcohol or drug abuse/dependancy were also excluded. The sample population included 15 males/14 females diagnosed with SZ and 24 age- and gender-matched general population controls ( 12 males/12 females) with no evidence for any psychiatric or neurological disorder in first-grade relatives (Table 1).

Table 1. Age descriptive statistics.

\begin{tabular}{|l|c|c|c|c|}
\hline Groups & $\boldsymbol{n}$ & Mean & SD & SE Mean \\
\hline SZ & 29 & 45.62 & 12.565 & 2.333 \\
\hline Controls & 24 & 46.38 & 12.673 & 2.587 \\
\hline
\end{tabular}

SD: standard deviation; SE: standard error; SZ: schizophrenia.

Blood Collection and RNA Isolation. Blood samples from the patients and control groups were collected in PAXgene Blood RNA collection tubes (PreAnalyticX GmbH, Hombrechtikon, Switzerland) that contain a reagent that lyses blood cells and immediately stabilizes intracellular RNA to preserve the gene expression profile. To reduce any potential bias in gene expression due to diurnal variation, blood was drawn in the morning, from all of the subjects. We used the PAXgene Blood miRNA Kit (PreAnalyticX) to extract total RNA from the blood samples [14]. Total RNA was then quantified by absorbance at A260 $\mathrm{nm}$ using Epoch Micro-Volume Spectrophotometer System (BioTek, Winooski, VT, USA) and the purity was estimated by the ratio A260/A280 $\mathrm{nm}$. The absorbance ratio of $260 \mathrm{~nm}$ and $280 \mathrm{~nm}$ (A260/A280) was between 1.93 and 2.1 for all samples included for further analysis. The RNA integrity was confirmed by non denaturing agarose gel electrophoresis, which was then stored at $-80^{\circ} \mathrm{C}$ until further analysis. The resulting RNA was treated with RNase-free DNase I (Promega BioSciences, San Luis Obispo, CA, USA) according to the manufacturer's protocol and checked for DNA contaminations prior to copy DNA synthesis step.PAGE 
Quantitative Reverse-Transcription Polymerase Chain Reaction (qRT-PCR) Analysis of FEZ1 mRNA Level. The qRT-PCR analyses were performed in at least three physically separate rooms in to order to reduce the chance for contamination. Copy DNA for the protein coding gene was synthesized from total RNA with oligo (dT) ${ }_{18}$ primer using RevertAid First Strand cDNA Synthesis Kit according to the assay protocol (Thermo Fisher Scientific, Waltham, MA, USA). Reverse transcription reactions contained $1 \mu \mathrm{g}$ of total RNA samples, $1 \mu \mathrm{L}$ oligo $(\mathrm{dT})_{18}$ primer and nuclease free water to a final volume of $12 \mu \mathrm{L}$, after incubation at $65{ }^{\circ} \mathrm{C}$ for $5 \mathrm{~min}$., we added $4 \mu \mathrm{L} 5 \mathrm{X}$ RT buffer, $1 \mu \mathrm{L}$ RiboLock RNase Inhibitor $(20 \mathrm{U} / \mu \mathrm{L})$ (Thermo Fisher Scientific), 2 $\mu \mathrm{L} 10 \mathrm{mM}$ dNTP Mix and $1 \mu \mathrm{L}$ RevertAid MMuLV Reverse Transcriptase (200 U/ $\mu \mathrm{L})$ (Thermo Fisher Scientific), to final volume of $20 \mu \mathrm{L}$. A relative gene expression method was employed to determine gene expression levels. The reactions were set up in duplicate in a 96-well format using the 7500 Real-Time PCR system (Applied Biosystems, Foster City, CA, USA) and Maxima SYBER Green/Rox qRT-PCR Kit (Thermo Fisher Scientific). Amplification of the single amplicon coresponding to the FEZ1 sequence was confirmed by monitoring the dissociation curve (melting curve analysis) and by agarose gel electrophoresis. The qRT-PCR forward and reverse primers for FEZI used in this study were 5'-GGG ACT GCA TGA GAC CAT GT-3' and 5'-TTG AGG GCT GTA GCC AGA CT-3', respectively [15]. B-actin $(A C T B)$ was used as an internal control for normalization. $A C T B$-specific qRT-PCR primers were as follows: forward 5'-AGT GTG ACG TGG ACA TCC GCA-3' and reverse 5'GCC AGG GCA GTG ATC TCC TTC T-3'.

After performing the qRT-PCR analysis, the $\mathrm{Ct}$ values were measured, different methods could be used to determine the expression level of the target gene in the test sample relative to the calibrator sample. Here, we used the Livak and Schmittgen method [16], also known as the comparative $2^{-\mathrm{Ct}}$ method. All the analyzed $\mathrm{SZ}$ samples showed mean $\mathrm{Ct}$ values range from 26.7 to 34.9 and mean control $\mathrm{Ct}$ values ranged from 28.0 to 31.3. The mean $\mathrm{Ct}$ values of the reference $A C T B$ gene for $\mathrm{SZ}$ samples ranged from 16.6 to 21.7 and the range for control samples from 16.3 to 18.0 , respectively.

First, for the normalization of the $\mathrm{Ct}$ values of the target genes to that of the reference gene, for both the test sample and the calibrator samples was made using the equation:

$$
\begin{aligned}
& \mathrm{Ct}_{\text {(test) }}=\mathrm{Ct}_{\text {(target, test) }}-\mathrm{Ct}_{\text {(reference, test) }} \text { - } \mathrm{Ct}_{\text {(calibrtator) }}=\mathrm{Ct}_{\text {(target, calibrator }} \\
& \mathrm{Ct}_{\text {(refe }}
\end{aligned}
$$

Second, normalization of the $\mathrm{Ct}$ of the test sample to the $\mathrm{Ct}$ of the calibrator was made using the equation:

$$
\mathrm{Ct}=\mathrm{Ct}_{\text {(test) }}-\mathrm{Ct}_{\text {(calibrator) }}
$$

Finally, calculation of the expression ratio with the following equation:

$2^{-\mathrm{Ct}}=$ normalized expression ratio.

The result obtained is the fold increase (or decrease) of the target gene in the test samples relative to the calibrator sample.

Statistical Analyses. All statistical calculations were performed using the Statistical Package for the Social Sciences (SPSS) software, version 20.0 (SPSS Inc., Chicago, IL, USA). Analysis of variance (ANOVA) $t$-test of $\mathrm{Ct}$ was used to examine differences in expression levels of FEZ1 mRNA across healthy controls and SZ subjects. A Spearman correlation analysis was also done for searching relation between expression levels and demographic characteristics of the tested individuals.

To investigate the characteristics of FEZ1 gene expression as potential diagnostic biomarkers in SZ patients, a ROC (receiver operating characteristic) curve was done and AUC (area under the ROC curve) was calculated. Statistical tests were two-sided with a $p$ value of 0.05 .

The STRING Pathway Analysis of FEZ1 Protein Interactions. The FEZ1 interaction data for each validated protein was obtained using the STRING database (version 9.0; http://string-db.org/) (Figure 1).

\section{RESULTS}

In order to identify whether a key DISCl (interacting partner gene) FEZ1 expression is altered in SZ patients we performed a detailed analysis of peripheral blood samples. To test this, we evaluated FEZ1 mRNA levels in SZ patients.

As the data were normally distributed, we performed two-tailed $t$-test. A statistically significant difference of FEZ1 mRNA level was revealed ( $p=$ 0.0068 ) (Figure 2). Relative quantification (RQ) calculations were done using the $2^{-\mathrm{Ct}}$ method [16]. The results clearly demonstrate down-regulation of FEZ1 mRNA levels in SZ patients. The data are presented 


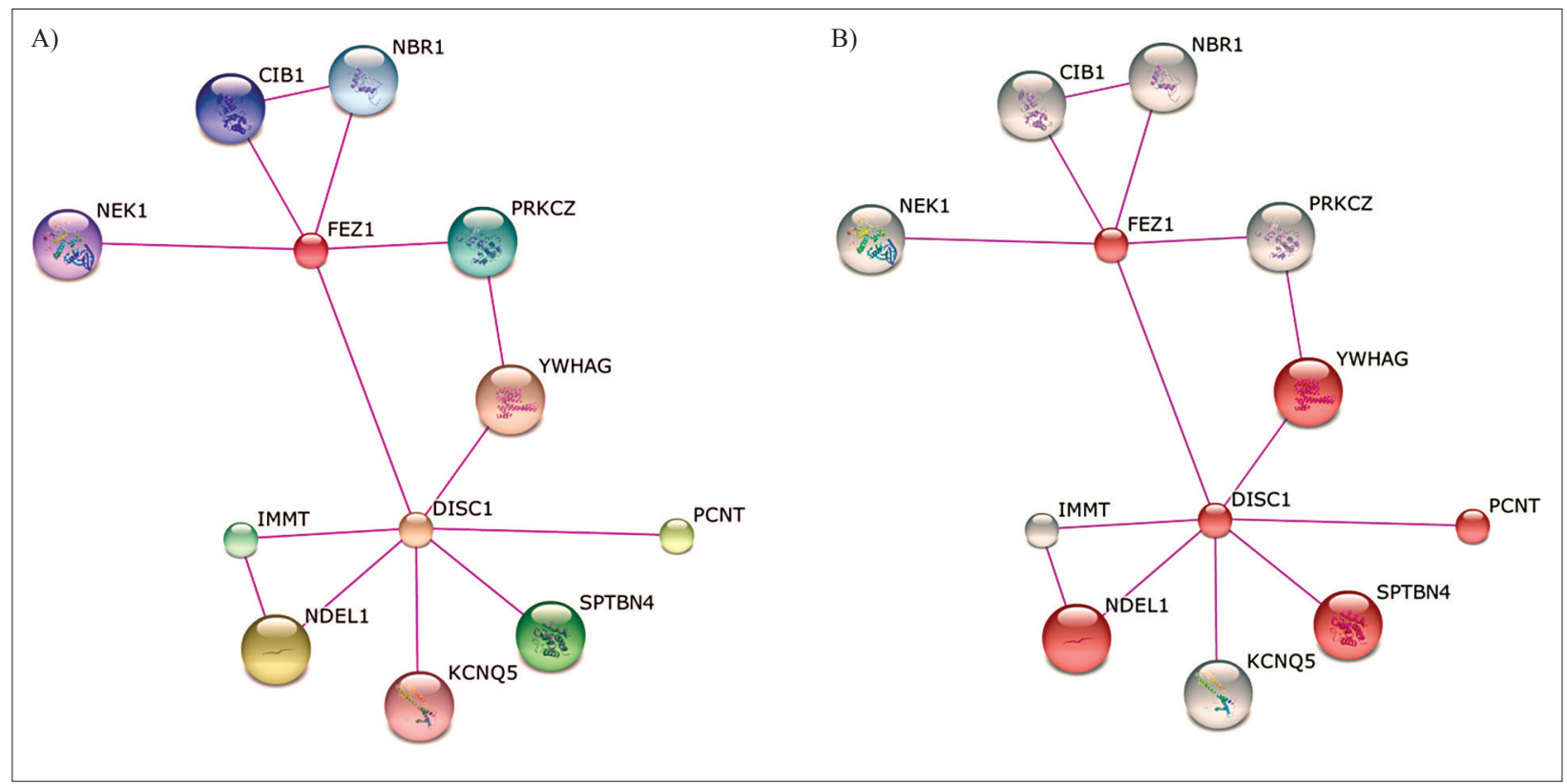

Figure 1. Network visualization of a key protein partners of FEZ1. A) The STRING screenshot shows an experimentally validated protein partners of FEZ1. B) Protein partners of FEZ1 (shown in red) in GO Biological Processes, term: neurogenesis.

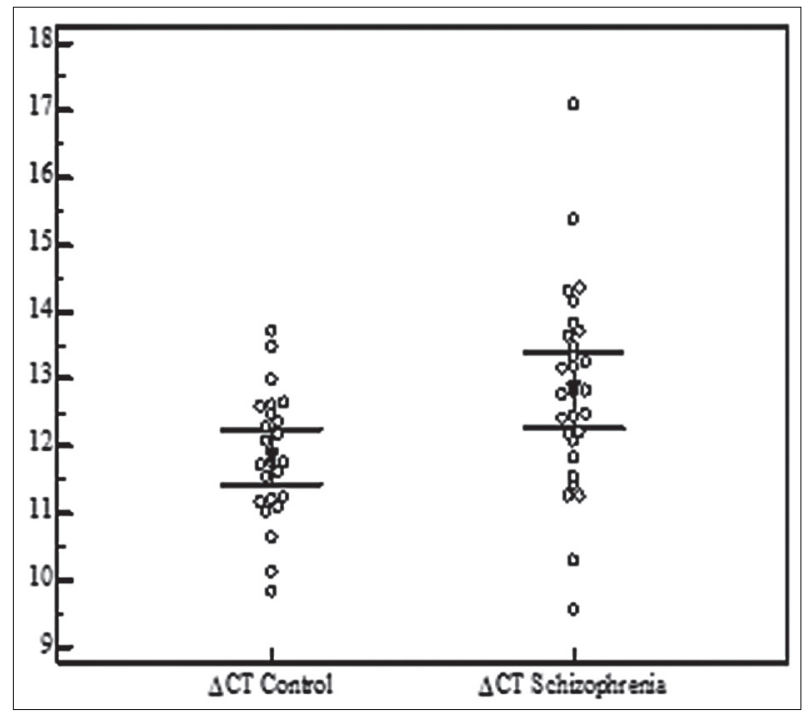

Figure 2. The figure shows the $\triangle \mathrm{Ct}$ values of FEZ1 expression across the analyzed SZ patients and healthy controls compared with the cycle in which they were obtained. The qRT-PCR procedure was used to determine relative quantification levels of FEZ1 mRNA (RQ FEZ1) in peripheral blood from 29 patient samples.

as the fold change in gene expression normalized to an endogenous reference gene and relative to the healthy control patients (Figure 3).

The observed differences in individual expression levels clearly demonstrate the heterogeneity in the expression profile of the analyzed gene that is in

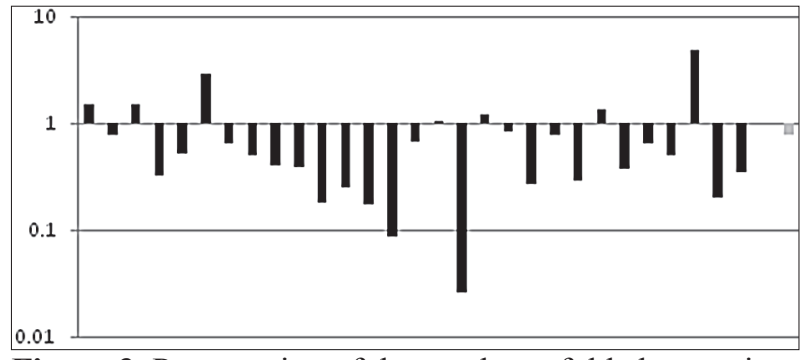

Figure 3. Presentation of the results as fold changes in gene expression normalized to an endogenous reference gene relative to the healthy control patients. Log transformed FEZ1 RQ of individual SZ subjects (in black), where 1 on the $y$-axis is average expression in controls. The average change of RQ level is shown in gray.

agreement with the results observed in inter-patient variation in the expression studies by other authors. The PCR amplification of the specific products corresponding to $A C T B$ and FEZ1 amplicons was confirmed by monitoring the dissociation curve (Figure 4).

The Spearman test did not manage to reveal any significant correlation with age and sex in the tested groups ( $p=0.65 / p=0.54)$. The ROC also displayed good characterization of state prediction with $\mathrm{AUC}=$ 0.728, $(p=0.0011)$ (Figure 5). All this was consistent with a key role of FEZ1 protein as interacting partner with DISC1 as SZ susceptibility genes (Figure 1A), and FEZ1 protein partners involved in neurogenesis 


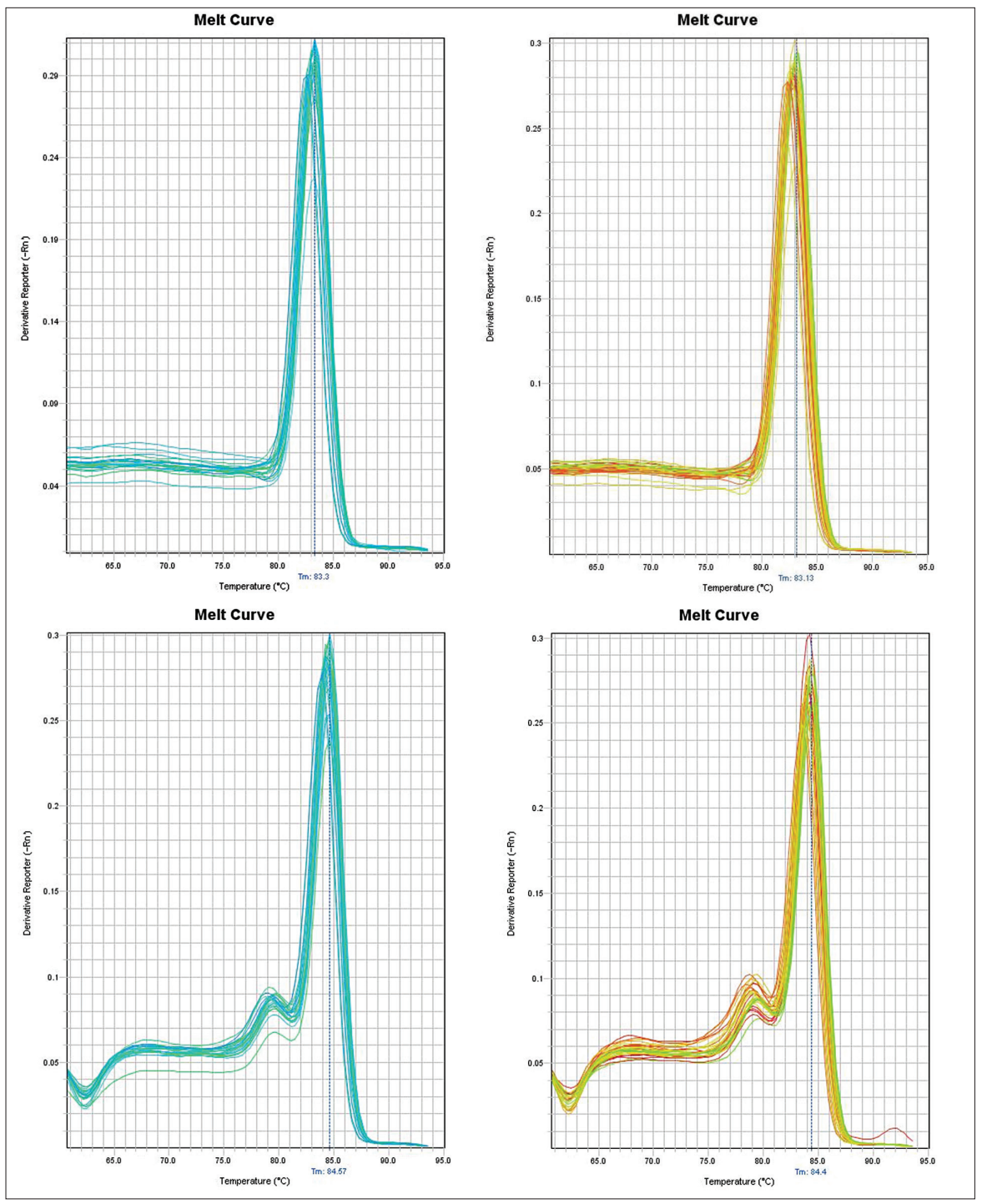

Figure 4. The qRT-PCR data showing a DNA melt profile result for amplification of the specific single product in qRT-PCR analysis. Specific single products corresponding to $A C T B$ in SZ and healthy control patients top panel and FEZ1 amplicons bottom panel, respectively, were confirmed by monitoring the dissociation curve (melting curve analysis). The melting dissociation, performed on FEZ1 and $A C T B$, allowed confirmation of the specificity of the amplifications. The melting temperatures of $F E Z 1$ amplicons were $84^{\circ} \mathrm{C} \pm 1{ }^{\circ} \mathrm{C}$, whereas $A C T B$ had a melting temperature of $83^{\circ} \mathrm{C} \pm 1^{\circ} \mathrm{C}$.

as shown in Gene Ontology (GO) Biological Processes $(p=0.063)$ (Figure 1B).

\section{DISCUSSION}

Despite the identification of numerous SZ susceptibility genes, the pathology of SZ still remains unknown. At the molecular level, a large number of potential DISC1 binding partners have been identified from a yeast two-hybrid screen [12], many of which are also involved in neurodevelopmental processes implicated in the patho-physiology of psychiatric disorders. Experiments with primates and rodents demonstrate that FEZ1 and DISC1 have overlapping temporal and spatial expression patterns $[17,18]$. Both proteins are expressed in the pyramidal neurons of the developing hippocampus, the cerebral neocortex and the olfactory bulb. Moreover, disruption of the DISC1/FEZ1 interaction inhibits DISC1-stimulated neurite outgrowth in PC12 cells 


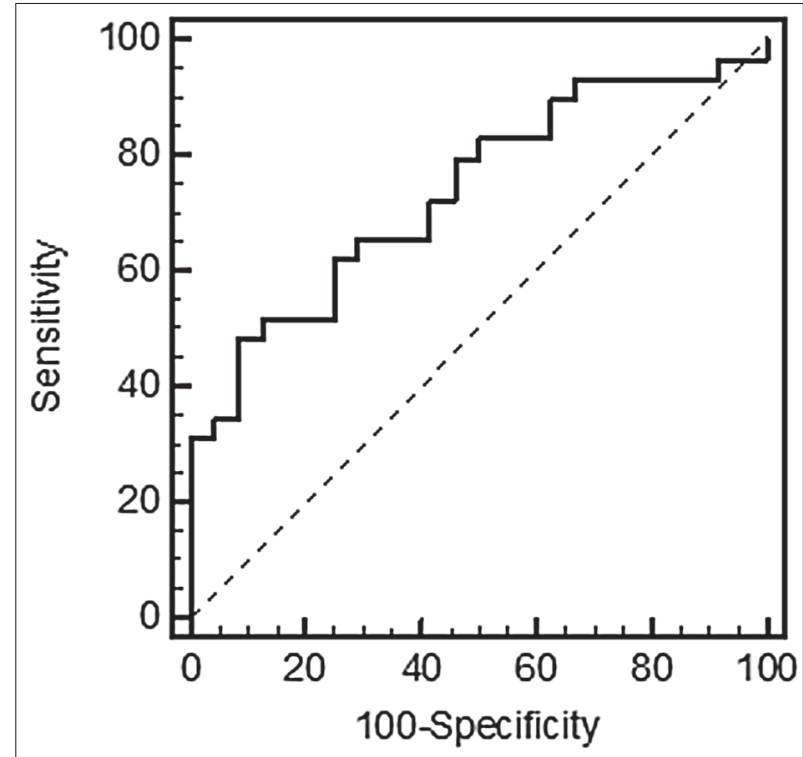

Figure 5. Performance of the FEZ1 prediction model. The ROC for FEZ1 expression was performed to evaluate the prediction accuracy. The dotted diagonal line represents random classification accuracy (AUC 0.5). The accuracy of FEZ1 as a single predictor was AUC 0.728 [95\% confidence interval (CI) minimum 0.589 / maximum 0.841; SE (standard error) 0.0698 ] with 55.2\% sensitivity and $87.5 \%$ specificity between schizophrenic and healthy controls subjects.

[8], thus, decreased FEZ1 levels could influence DISC1-stimulated functions.

On the one hand, this study is the first describing a FEZ1 gene expression change in peripheral blood of patients with SZ; on the other hand, this change corresponds to the down-regulation in prefrontal cortex and hippocampus of schizophrenic patients [9]. Due to the fact that FEZ1 interacts with $D I S C 1$, a susceptibility gene for major mental disorders to synergistically regulate dendritic growth of newborn neurons in the adult mouse hippocampus. We assume that any interaction related to another FEZ1 partner would be compromised or at least influenced due to changes in the expression of the FEZ1 transcript that would change the level of translated protein and its involvement in complexes associated with susceptibility for SZ functions (Figure 1).

Thus, our results provide support for a model of SZ pathogenesis that includes the regulatory effects on FEZ1 gene expression in peripheral blood specific for patients with exacerbation of SZ. One obvious limitation of previous expression studies is the use of human postmortem brain tissue for quantitative analyses of gene expression profile, primarily because postmortem brain tissue from SZ patients is extremely rare and highly prized. Additionally, identification of gene expression profile can be complicated by a variety of confounding factors such as $\mathrm{pH}$, drugs, cause of death, etc. In contrast, in attempts to overcome these limitations, we used RNA storage and extraction systems that block and preserve RNA for downstream expression study, so that the expression level that we identified really reflects the current physiological state of the analyzed patients. All these changes in expression levels probably are not due to gene polymorphisms, as such was not detected in a large SZ cohort [19], but can be attributed to various epigenetic mechanisms that alter distinct molecular pathways. As hypomethylation was observed in the exonic region of $H T R 2 A$ and $M B-C O M T$ promoters in the DNA derived from saliva in SZ [20], it is possible that epigenetic factors leading to down-regulation of FEZ1 in schizophrenic brains may also reflect in peripheral blood and result in the reduction of the expression in these tissues. However, additional studies applying RNA sequencing analysis for identifying peripheral blood-based biomarkers that could represent brain expression and epigenetic aberrations remain a key step in implication of these findings in pathogenesis, diagnosis and future therapy.

\section{ACKNOWLEDGMENTS}

We gratefully thank all the probands and their families who were enrolled in this study and all the control subjects who donated blood samples.

Declaration of Interest. This study was supported by MU - Plovdiv Project No. HO - 4/2011. The authors report no conflicts of interest. The authors alone are responsible for the content and writing of this article.

\section{REFERENCES}

1. Perala J, Suvisaari J, Saarni SI, Kuoppasalmi K, Isometsä E, Pirkola S, et al. Lifetime prevalence of psychotic and bipolar I disorders in a general population. Arch Gen Psychiatry. 2007; 64(1): 19-28. 
2. Ng MY, Levinson DF, Faraone SV, Suarez BK, DeLisi Le, Arinami T, et al. Meta analysis of 32 genome-wide linkage studies of schizophrenia. Mol Psychiatry. 2009; 14(8): 774-785.

3. Shi J, Levinson DF, Duan J, Sanders AR, Zheng Y, Pe'er I, et al. Common variants on chromosome $6 \mathrm{p} 22.1$ are associated with schizophrenia. Nature. 2009; 460(7256): 753-757.

4. Stefansson H, Ophoff RA, Steinberg S, Andreassen OA, Cichon S, Rujescu D, et al. Common variants conferring risk of schizophrenia. Nature. 2009; 460(7256): 744-747.

5. Purcell SM, Wray NR, Stone JL, Visscher PM, O’Donovan MC, Sullivan PF, et al. Common polygenic variation contributes to risk of schizophrenia and bipolar disorder. Nature. 2009; 460(7256): 748-752.

6. Vawter MP, Ferran E, Galke B, Cooper K, Bunney WE, Byerley W, et al. Microarray screening of lymphocyte gene expression differences in a multiplex schizophrenia pedigree. Schizophr Res. 2004; 67(1): 41-52.

7. Kuzman MR, Medved V, Terzic J, Krainc D. Genome-wide expression analysis of peripheral blood identifies candidate biomarkers for schizophrenia. J Psychiatr Res. 2009; 43(13): 1073-1037.

8. Miyoshi K, Honda A, Baba K, Taniguchi M, Oono K, Fujita T, et al. Disrupted-In-Schizophrenia 1, a candidate gene for schizophrenia, participates in neurite outgrowth. Mol Psychiatry. 2003; 8(7): 685-694.

9. Lipska BK, Peters T, Hyde TM, Halim N, Horowitz C, Mitkus T, et al. Expression of DISC1 binding partners is reduced in schizophrenia and associated with DISC1 SNPs. Hum Mol Genet. 2006; 15(8): 1245-1258.

10. Sakae N, Yamasaki N, Kitaichi K, Fukuda T, Yamada M, Yoshikawa H, et al. Mice lacking the schizophrenia-associated protein FEZ1 manifest hyperactivity and enhanced responsiveness to psychostimulants. Hum Mol Genet. 2008; 17(20): 3191-3203.

11. Moens LN, de Rijk P, Reumers J, van den Bossche MJ, Glassee W, de Zutter S, et al. Sequencing of DISC1 pathway genes reveals increased burden of rare missense variants in schizophre- nia patients from a Northern Swedish population. PLoS ONE. 2011; 6(8): e23450. doi: 10.1371/ journal.pone. 0023450 .

12. Chubb JE, Bradshaw NJ, Soares DC, Poeteous DJ, Millar JK. The DISC locus in psychiatric illness. Mol Psychiatry. 2008; 13(1): 36-64.

13. Abdolmaleky HM, Cheng KH, Faraone SV, Wilcox M, Glatt SJ, Gao F. Hypomethylation of MB-COMT promoter is a major risk factor for schizophrenia and bipolar disorder. Hum Mol Genet. 2006; 15(21): 3132-3145.

14. Wang J, Robinson JF, Khan HM, Carter DE, McKinney J, Miskie BA, et al. Optimizing RNA extraction yield from whole blood for microarray gene expression analysis. Clin Biochem. 2004; 37(9): 741-744.

15. Yu Z, Ono C, Kim HB, Komatsu H, Tanabe Y, Sakae N, et al. Four mood stabilizers commonly induce FEZ1 expression in human astrocytes. Bipolar Disord. 2011; 13(5-6): 486-499.

16. Livak KJ, Schmittgen TD. Analysis of relative gene expression data using real-time quantitative PCR and the $2(-\Delta \Delta \mathrm{C}(\mathrm{T}))$ method. Methods. 2001; 25(4): 402-408.

17. Austin CP, Ky B, Ma L, Morris JA, Shughrue PJ. Expression of disrupted-in-Schizophrenia-1, a schizophre-nia-associated gene, is prominent in the mouse hippocampus throughout brain development. Neuroscience. 2004; 124(1): 3-10.

18. Honda A, Miyoshi K, Baba K, Taniguchi M, Koyama Y, Kuroda S, et al. Expression of fasciculation and elongation protein zeta-1 (FEZ1) in the developing rat brain. Brain Res Mol Brain Res. 2004; 122(1): 89-92.

19. Koga M, Ishiguro H, Horiuchi Y, Albalushi T, Inada $\mathrm{T}$, Iwata $\mathrm{N}$, et al. Failure to confirm the association between the FEZ1 gene and schizophrenia in a Japanese population. Neurosci. Lett. 2007; 417(3): 326-329.

20. Nohesara S, Ghadirivasfi M, Mostafavi S, Eskandari MR, Ahmadkhaniha $\mathrm{H}$, Thiagalingam $\mathrm{S}$, et al. DNA hypomethylation of MB-COMT promoter in the DNA derived from saliva in schizophrenia and bipolar disorder. J Psychiatr Res. 2011; 45(11): 1432-1438. 
